\title{
Zur Problematik
}

\section{der intraurbanen Subzentrenforschung}

\section{Begriffliches und Methodisches}

Unter intraurbanen (oder innerstädtischen) Subzentren sollen die außerhalb des Hauptgeschäftszentrums (der City) liegenden Geschäftszentren verstanden werden. ${ }^{1)}$

Wir entsprechen dabei mit unserem Begriffsverständnis dem englischen Sprachgebrauch, der alle Zentren außerhalb der City als «subsidiary busineß districts» bezeichnet (Lit. 5, p. 310ff).

Erste Identifikationen von Mustern städtischer Subzentren mit hierarchischem Aufbau erfolgten auf rein deskriptivem Wege durch PROUDFOOT (Lit.13) und BURNS (Lit.4). CAROL übertrug das zentralörtliche Schema CHRISTALLERS auf die Stadt (Lit. 6, 7). Er stellte am Beispiel der Stadt Zürich ein hierarchisches System von innerstädtischen Zentralen Orten über einem «Muster von Läden um die Ecke» fest. Nach ihrer flächenwerksamen Versorgungsfunktion, den Unterschieden im angebotenen Sortiment, der Häufigkeit der Bedarfsdeckung und nach dem Indikator verschiedener Fußgängerdichten als $\mathrm{Ma}$ für die Intensität der Kundenfrequenz unterschied CAROL außerhalb des Hauptgeschäftszentrums Regionalzentren, Nachbarschaftszentren und Lokalzentren mit verschieden gewichteten Funktionen im städtischen Versorgungsprozeß.

Drei Versuche zur Ermittlung einer Hierarchie innerstädtischer Zentren sind von ihrem methodischen Ansatz her erwähnenswert, obwohl sie nicht über die formale Identifikation hinausführten: GARNER (Lit. 8) ermittelte eine hierarchische Gruppierung der Subzentren in Chicago mit Hilfe von Bodenpreisprofilen in den Geschäftsstraßen. JOHNSTON (Lit. 10) konstruierte ein achtstufiges Zentrenmodell für Melbourne mit Hilfe der Wohndichte, des sozio-ökonomischen Status und des Arealalters im städtischen Entwicklungsprozeß. JONES (Lit. 11) bildete in der Stadt Edinburgh aus den Gesamtladenflächen und der Anzahl ausgewählter Geschäftstypen pro Subzentrum sieben Klassen für eine innerstädtische Zentrenhierarchie. Mit einem konsequenten quantitativen Ansatz ermittelte SEDLACEK (Lit. 14) in Münster eine hierarchische Gliederung intraurbaner Zentralorte aus dem Versorgungswert der Ladengeschäfte, ${ }^{2)}$ dem branchenrelativen Arbeitskräftebesatz und den mittleren Kundenfrequenzen. Im weiteren charakterisierte er die Subzentren quantitativ bezüglich des Auftretens bestimmter Wirtschaftsgruppen des Einzelhandels und nach Frequenztypus.

Zusammenfassend können wir feststellen:

Die Analysen von Subzentren erfolgten

- mit empirisch-deskriptiven Methoden (PROUDFOOT, BURNS)

- durch Verwendung von Indikatoren und deren teilweise quantitative Gewichtung (CAROL, GARNER, JOHNSTON, JONES)

- mit Methoden der quantitativen Verarbeitung ausgewählter Daten (Sedlacek).

Die dabei angesetzten Methoden dienen in der Mehrzahl der Fälle der Ermittlung formaldeskriptiver Subzentren-Hierarchien, welche als statisch-geometrische Modelle bildhaft gemacht werden. Die funktionale Einordnung der Subzentren in den städtischen Versorgungsprozeß erhält in der Regel kein oder nur wenig Gewicht; am weitgehendsten wird dieser Aspekt durch CAROL (Lit.6) berücksichtigt. Die Arbeit von ABELE/ LEIDLMAIR (Lit. 1) steht insofern am Rande unserer Betrachtungen, als sie im Hauptgeschäftszentrum und in den höchstwertigen Subzentren wirtschaftsgeographische und sozioökonomische Strukturen analysiert.

\section{Die Praxisrelevanz intraurbaner Subzentrenforschung}

Die Erarbeitung städtischer Zentrenhierarchien war in den Fünfziger- und Sechzigerjahren insofern bedeutungsvoll, als damit Modelle der räumlichen Verteilung und Gewichtung von Hauptund Subzentren der Stadtplanung Entscheidungshilfen leisten konnten. Auf Erhebungen im Auftrage des Planungsamtes der Stadt Zürich beispielsweise ist die Publikation von CAROL (Lit. 1) aufgebaut.

Die Notwendigkeit planerischer Sicherung und

Prof. Dr. Erich Bugmann, Forschungsstelle für Wirtschaftsgeographie und Raumplanung an der Hochschule St. Gallen, General-Guisan-Straße 70, 9010 St. Gallen 
Förderung der Hauptgeschäftszentren ist heute kaum umstritten. Denn sie sollen trotz der Konkurrenz von Einkaufszentren (Shopping Centers) in Stadtnähe und auf der Grünen Wiese die Versorgung eines grösseren städtischen Umlands mit Gütern des gehobenen und gelegentlichen Bedarfs sicherstellen; sie haben aber auch ein engeres bis weiteres Stadtgebiet mit Gütern des Grundbedarfs und des periodischen Bedarfs zu versorgen.

Die Bedeutung der Subzentren wird mit wachsenden Marktanteilen der Grossverteiler inner- und ausserhalb des Stadtgebiets und mit der Zunahme des motorisierten Einkaufsverhaltens mehr und mehr in Frage gestellt. Nachdem das Ladensterben von den Einzelläden auf die Subzentren überzugreifen begonnen hat, muß man sich fragen: Ist es überhaupt noch sinnvoll, in städtischen Flächennutzungsplänen Raum für Subzentren zu reservieren? - Wenn ja, wie sind ihre räumliche Verteilung und ihre potentiellen Wirkungsbereiche im Stadtgebiet einzuordnen? - Zur Lösung dieser Fragen sind einerseits problemgerichtete Bestandesaufnahmen und ständige Überwachung mit periodischen Datenerhebungen erforderlich. Andrerseits müßen normative Anforderungen an die Subzentren und ihre Funktionen im städtischen Versorgungsprozess gestellt werden.

\section{Ein Vorschlag für eine operationale planungspraxisbezogene Arbeitsmethode}

Im Anschluß an Arbeiten im Hauptgeschäftszentrum der Stadt St. Gallen (Lit.3) wurden im Rahmen der wirtschaftsgeographischen Praktika der Hochschule St. Gallen in den Jahren 1975 und 1976 Daten in den Stadtquartieren erhoben und nach verschiedenen Methoden ausgewertet. Die Datensätze wurden im Jahre 1977 überprüft, ergänzt und die Datenträger kartographisch genau lokalisiert. Damit standen für die empirische Überprüfung eines praktikablen methodischen Ansatzes Basiswerte zur Verfügung.

Unser Verfahren setzt sich aus folgenden Arbeitsschritten zusammen:

1. Identifikation und Abgrenzung der Subzentren, ihre Differenzierung und Gewichtung nach der Art der Bedarfsdeckung.

2. Bestimmung von

- Versorgungsqualität, Versorgungsbereichen und Versorgungsgrad im Stadtraum.

- Einzelhandelskapazität und Auslastungsgrad der Subzentren,

insbesondere auf der Stufe der Deckung des Grundbedarfs.

3. Vergleich von Subzentren und zugeordneten Versorgungsbereichen bezüglich

- Subzentrumsattraktivität
- sozio-ökonomischen Quartierstrukturen

- aktuellem Einkaufsverhalten der Bevölkerung.

4. Planerische Freihaltung oder Sicherung in den städtischen Richt- und Nutzungsplänen.

5. Dauernde Überwachung und periodische Datenüberprüfung im Hinblick auf mögliche oder notwendige Revisionen in den Flächennutzungsplänen.

Die vorgeschlagenen Arbeitsschritte werden nachfolgend erläutert und diskutiert.

\subsection{Identifikation und Abgrenzung der Sub-} zentren, ihre Differenzierung und Gewichtung nach Stufen der Bedarfsdeckung.

\subsubsection{Identifikation und Abgrenzung}

Wesentliches Merkmal eines Subzentrums ist die Konzentration von Angebotsstandorten. Sie schließt in sich eine Reduktion der Wegzeiten beim Einkauf. Ein Subzentrum wird demnach definiert als eine räumliche Agglomeration von mindestens drei Geschäften des Einzelhandels, wobei Großverteilerfilialen oder Diskountgeschäfte je nach ihren Angeboten zwei oder mehreren Einzelgeschäften gleichgesetzt werden können. Die Distanzen zwischen den Einzelgeschäften sollen folgende Minimalwerte nicht überschreiten: im reinen Fußgängerbereich $50 \mathrm{~m}$, über Nebenstraßen $40 \mathrm{~m}$ und über Hauptstraßen $20 \mathrm{~m}$. Mit diesen normativ gesetzten Distanzwerten wird in vereinfachter Form das Prinzip der Geschäftsdichte im Sinne von ABELE/LEIDLMAIR (Lit. 1, p.41-42) und SEDLACEK (Lit. 14, p. 13-20) einbezogen. Wohl können auch Einzelgeschäfte Teile des Stadtraumes mit Gütern versorgen. Es fehlen ihnen aber die planerisch relevante Zentrumsfunktion und die Mindestgröße für eine Flächenausscheidung in der. Nutzungsplanung.

Abb. 1 zeigt vorgenommene Subzentrenabgrenzungen an den Beispielen Lachen, Neudorf, Rotmonten, Abb. 3 die Lage der identifizierten Subzentren im Stadtgebiet von St. Gallen.

\subsubsection{Differenzierung der Geschäfte nach \\ Bedarfsstufen}

Wir verwenden in ähnlicher Weise wie SEDLACEK (Lit. 5, p.68-70), indikatorartige Daten von Geschäften des Einzelhandels. Miteinbezogen werden aber solche von Geschäften mit einem mehr oder weniger starken Dienstleistungscharakter, welche im Publikumsverkehr sowohl reale Güter als auch Dienstleistungen verkaufen, wie z.B. Banken, Apotheken, Postfilialen, Cafés und Reisebüros. Die in der Tabelle 1 aufgestellte Branchengliederung faßt verwandte Geschäftstypen zusammen und garantiert damit gute Über- 


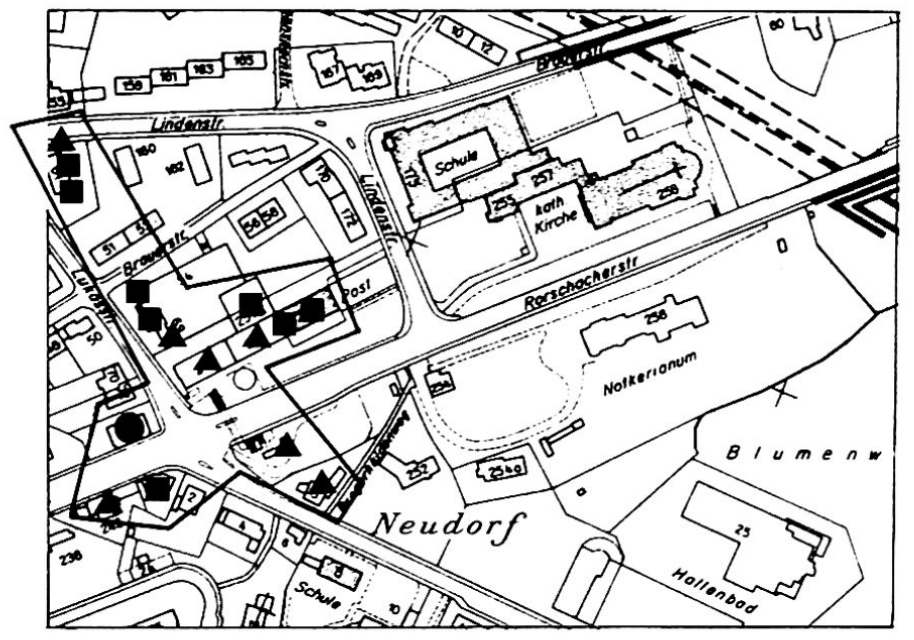

Abb. 1:

Beispiele der Subzentrumsabgrenzung in der Stadt St. Gallen

Geschäfte mit vorwiegendem Angebot für:

- Grundbedarf

- Periodischer Bedarf

- Aperiodischer Bedarf

Branchenübergreifend (kommt nicht vor)
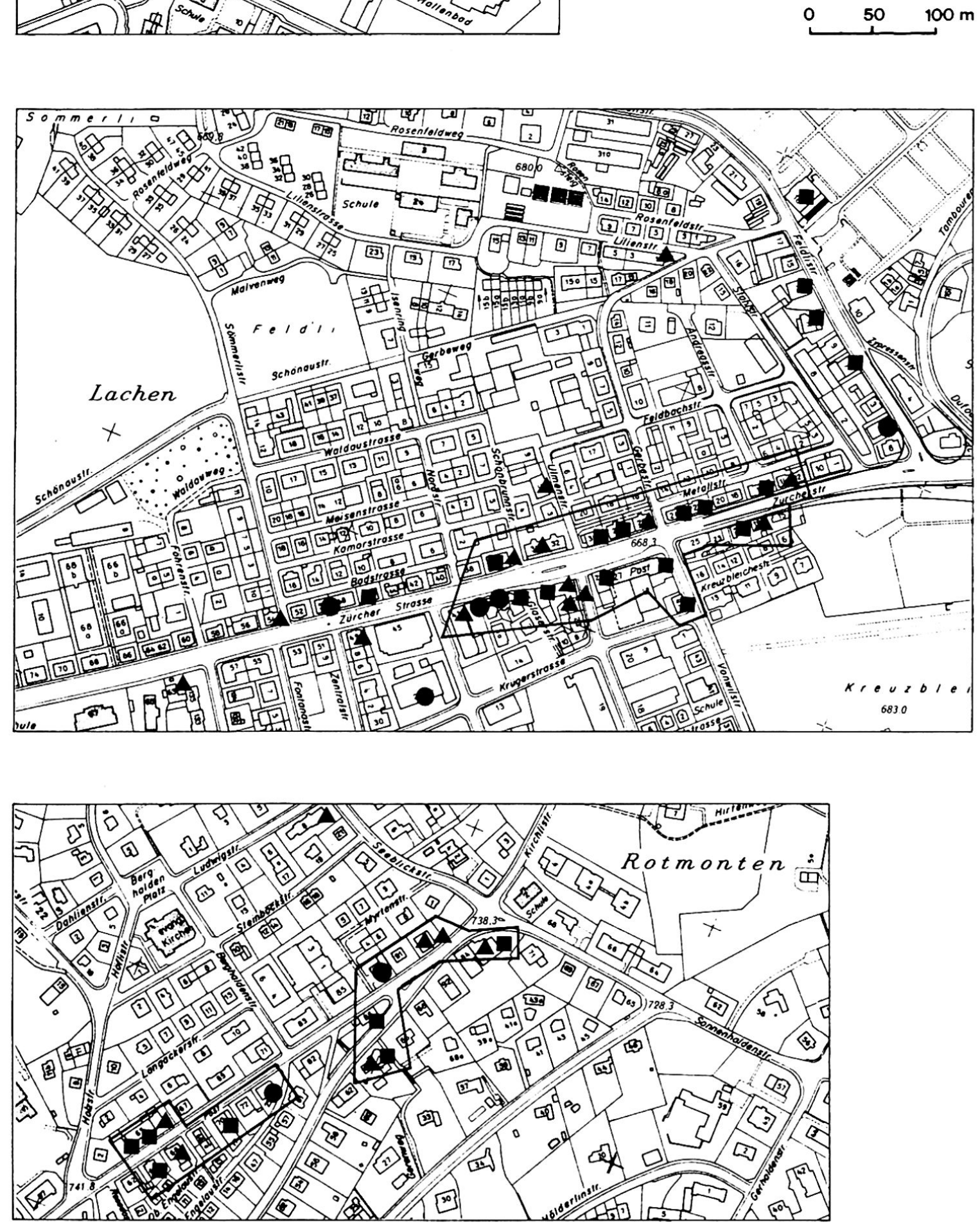
sichtlichkeit. Die Zuordnung der Branchen zu bestimmten Typen der Bedarfsdeckung hat einen generellen Charakter. Im Einzelfall kann jedes Geschäft der branchentypischen oder einer andern Stufe der Bedarfsdeckung zugewiesen oder bei Fehlen von Einzelhandelsfunktionen (z.B. bei Restaurants) ausgeschaltet werden.

Tabelle 1:

Branchengliederung und Art der Bedarfsdeckung

Die Bedarfsdeckung erfolgt in der Regel:

$\mathrm{G}=$ für den Grundbedarf (täglichen Bedarf)

$\mathrm{p}=$ für den periodischen Bedarf

$\mathrm{a}=$ für den aperiodischen Bedarf

\begin{tabular}{ll}
\hline Branchen & $\begin{array}{l}\text { Bedarfs- } \\
\text { deckung für: }\end{array}$ \\
\hline
\end{tabular}

1 Lebens- und Genußmittel G

2 Filialen von Großverteilern G

3 Bekleidung, Textilien, Lederwaren

4 Haushalt- und Eisenwaren

$\mathrm{p}$

a

5 Zeitungen, Zeitschriften, Bücher, Papeterien, Kioske G (p)

6 Drogerie, Apotheke

7 Blumen

8 Uhren, Schmuck

9 Foto, Optik

10 Hobby, Sport, Farben, Gartengeräte

11 Elektrowaren, Radio, TV, Autozubehör

12 Möbel, Teppiche, Tapeten, Innendekoration

$\mathrm{p}$

a

(p) $\mathrm{a}$

a

a

a

13 Kauf- und Warenhäuser, Grossverteiler mit erheblichem

Nonfood-Anteil

14 Café, Restaurant

(G)

$\mathrm{p} \quad \mathrm{a}$

p a

15 Coiffeur, Kosmetika

$\mathrm{p}$

Chemische Reinigung

$\mathrm{p}$

Bankfiliale

$\mathrm{p}$

18 Postfiliale

$\mathrm{p}$

19 Reisebüro

$\mathrm{p} \quad \mathrm{a}$

\subsubsection{Gewichtung der Subzentren nach Bedarfsstufen}

Schon eine Kartierung nach Branchen und Bedarfsstufen (Beispiele in Abb.1) läßt die vorwiegende Bedeutung jedes einzelnen Subzentrums im städtischen Versorgungsprozess abschätzen. Eine weitergehende Gewichtung ist für den innerstädtischen Vergleich jedoch wünschenswert.

Die von uns in St. Gallen erhobenen Daten wurden zur Ermittlung eines Maßes für die Zentralität der Subzentren mit der Methode von SEDLACEK
(Lit.14) durchgerechnet. Dabei zeigte sich, daß eine generelle Verschmelzung der eingegebenen Daten zum Transparenzverlust führt. Weiter ergaben sich bei Anwendung dieser Methode auf die Verhältniße in der Stadt St.Gallen auffällige Verzerrungen. So resultierte beispielsweise aus der Eingabe der mittleren Frequenz eine ungebührliche Aufwertung der - wöchentlich mehrmals frequentierten - vorwiegend für die Grundbedarfsdeckung ausgelegten kleineren Zentren. Bei Gleichbehandlung der Versorgungswerte von Geschäften bestimmter Branchen 2) erhielten Subzentren mit singulären Branchen (z. B. Photogeschäften, Reisebüros) eine unverhältnismäßig hohe Gewichtung. Wenn wir die Arbeitskräfte der in einem Zentrum vorhandenen Geschäfte aufsummieren, erhalten wir den Arbeitskräftebesatz, der in Kolonne 2 von Tabelle 2 festgehalten ist. Diese Zahlenwerte könnten durchaus für eine Hierarchiebildung und die Erstellung einer Zentrenrangordnung verwendet werden. Die im Publikumsverkehr eingesetzten Arbeitskräfte sind Indikatoren für erzielte $\mathrm{Ge}$ schäftsumsätze. Die Wertschöpfung ist allerdings bei verschiedenen Branchen pro Arbeitskraft verschieden 3). Diese Differenzen in der Wertschöpfung sind bereits wesentlich geringer bei Geschäften auf der Stufe der Grundbedarfsdeckung. Mit dem Arbeitskräftebesatz dieser Branchen läßt sich jedoch höchstens etwas über die Qualität der Grundversorgung aussagen (Kolonne 3 in Tabelle 2).

Aus Tabelle 2 ist ersichtlich, daß sich sowohl unter Verwendung der Methode SEDLACEK (Kolonne 1) als auch mit Hilfe des aufgerechneten Arbeitskräftebesatzes (Kolonne 2) eine Rangordnung der Subzentren erstellen läßt. Die Rangfolgen sind in einigen Positionen übereinstimmend. Eine Hierarchiebildung läßt sich grundsätzlich nach empirischen, mathematisch-statistischen oder funktionellen Gesichtspunkten vornehmen. Aus der Kenntnis der örtlichen Verhältnisse scheint die Zuweisung des Zentrums Großacker zu einer höchsten und jene der letzten fünf Zentren der Liste zu einer tiefsten Klasse vertretbar; eine Aufgliederung der Kerngruppe ist mit empirischen Maßstäben nicht möglich. Zum selben Ergenis muß der Versuch einer mathematisch-statistischen Klassenbildung führen. Es ist darum sinnvoll, eine Gliederung der Subzentren nach ihrer funktionellen Bedeutung im städtischen Versorgungsprozeß vorzunehmen.

Städtische Subzentren dienen zum Teil der Grundversorgung, zum Teil der Versorgung mit Gütern des periodischen und aperiodischen Bedarfs. Wir spalten in einem ersten Arbeitsschritt die Subzentren nach dem Geschäfts- und Arbeitsplatzbesatz der drei Bedarfsgruppen auf. (Abb. 1, 2). Um den besonderen Charakter von Großverteiler- und Warenhausfilialen gerecht werden zu können, wird für 
Abb. 2

Die Ausstattung der Subzentren in St. Gallen

Anzahl Arbeitskräfte pro Betrieb und

Bedarfsstufe (1mm = 1 Arbeitskraft)

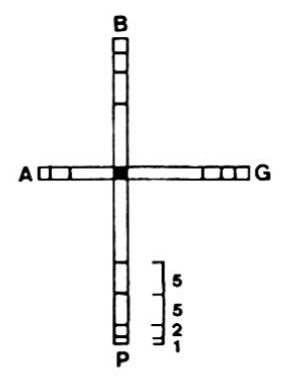

G Grundbedarf

P Periodischer Bedarf

A Aperiodischer Bedarf

B Branchenübergreifendes Sortiment

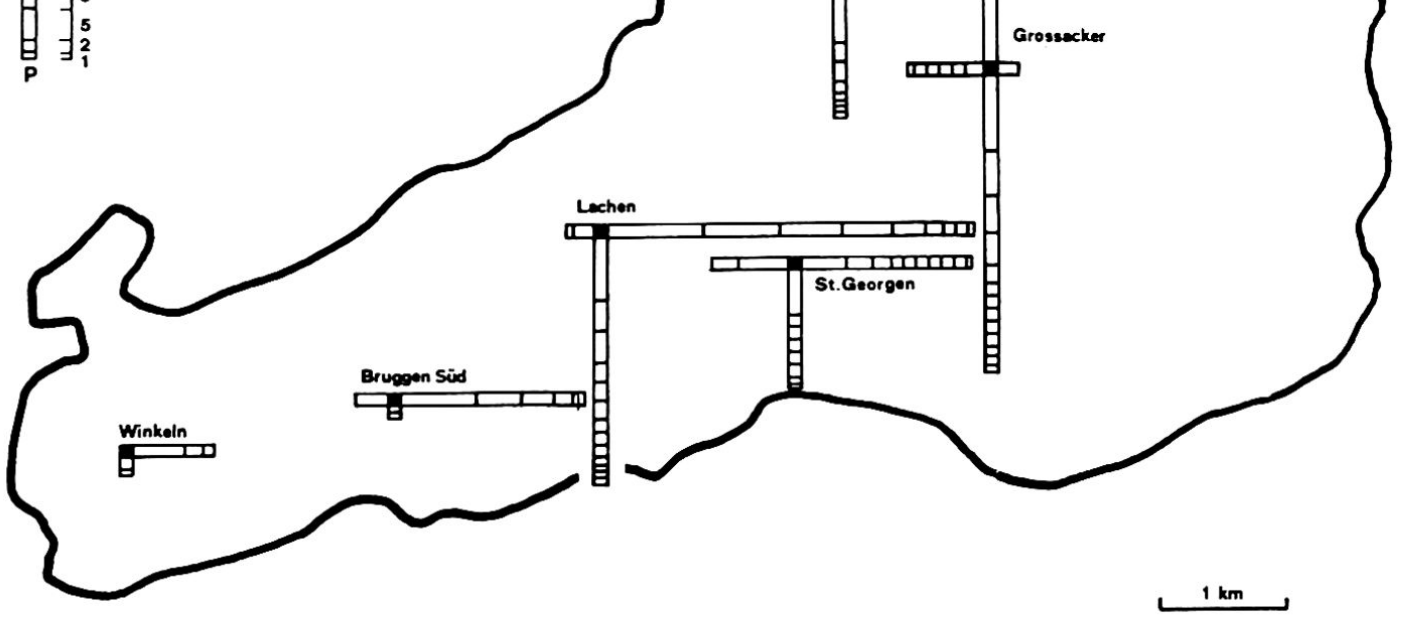

Abb. 3 Lagegerechter Situationsplan der Subzentren in St. Gallen (mit Bewilligung des Bundesamtes für Landestopographie)

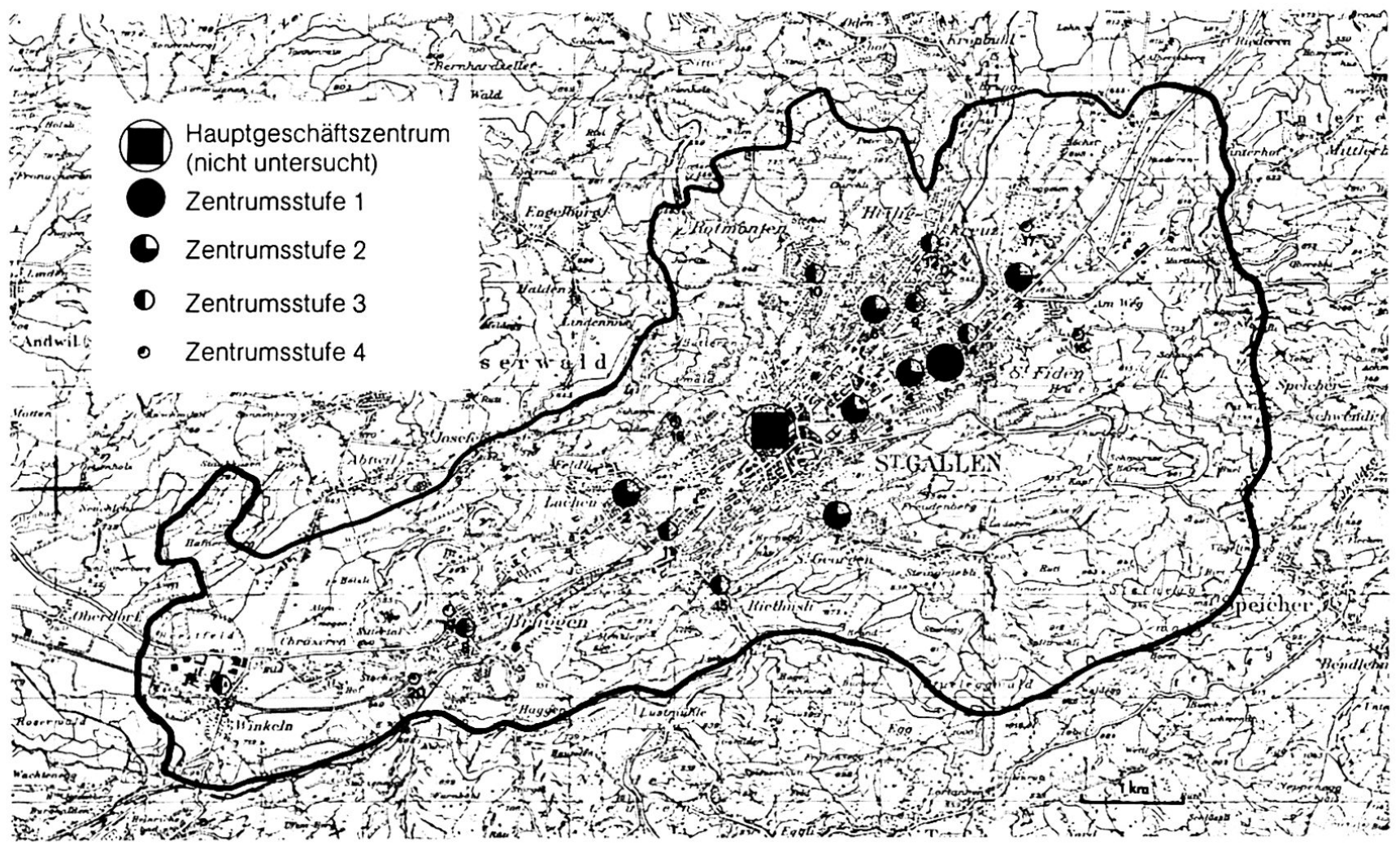


diese Geschäftsform eine zusätzliche Kategorie «branchenübergreifendes Angebot» verwendet. Die Graphiken in Abb. 2 lassen bereits gewisse Interpretationen im Hinblick auf die Auslegung einzelner Subzentren zu.

Eine weitergehende Typisierung hat nun nach der Art der Bedarfsdeckung zu erfolgen. Da die Qualität der Grundversorgung (der Versorgung mit Gütern des täglichen Bedarfs) eine zentrale Fragestellung ist, verwenden wir nachstehendes Schema, welches primär auf dem Angebot der Güter des täglichen Bedarfs aufgebaut ist 4) (vgl. auch Abb. 3).

\begin{tabular}{|c|c|c|}
\hline $\begin{array}{l}\text { Zentrums- } \\
\text { stufe }\end{array}$ & $\begin{array}{l}\text { Angebot auf dem } \\
\text { Niveau der Grund- } \\
\text { versorgung }\end{array}$ & $\begin{array}{l}\text { Angebot für höheren } \\
\text { Bedarf }\end{array}$ \\
\hline 1 & $\begin{array}{l}\text { sichergestellt } \\
\text { keine Branchen } \\
\text { konkurrenz }\end{array}$ & $\begin{array}{l}\text { fehlend oder } \\
\text { unbedeutend }\end{array}$ \\
\hline 2 & $\begin{array}{l}\text { sichergestellt } \\
\text { mit Branchen- } \\
\text { konkurrenz }\end{array}$ & $\begin{array}{l}\text { fehlend oder } \\
\text { unbedeutend }\end{array}$ \\
\hline 3 & $\begin{array}{l}\text { sichergestellt } \\
\text { mit Branchen- } \\
\text { konkurrenz }\end{array}$ & $\begin{array}{l}\text { für periodischen } \\
\text { Bedarf vorhanden } \\
\text { für aperiodischen } \\
\text { Bedarf fehlend } \\
\text { oder unbedeutend }\end{array}$ \\
\hline 4 & $\begin{array}{l}\text { sichergestellt } \\
\text { mit Branchen- } \\
\text { konkurrenz }\end{array}$ & $\begin{array}{l}\text { für periodischen } \\
\text { und aperiodischen } \\
\text { Bedarf angeboten, } \\
\text { z.T. mit Branchen- } \\
\text { konkurrenz }\end{array}$ \\
\hline
\end{tabular}

Dieser Typisierung liegen folgende Überlegungen zugrunde:

- Notwendige Bedingung für den Zentrumscharakter ist (über eine minimale Anzahl von Geschäften hinaus) das Angebot einer vollständigen Grundversorgung.

- Sind zwei oder mehrere Geschäfte der gleichen Branche innerhalb einer Bedarfsstufe auf engem Raume vorhanden, so erhöht sich die Qualität des Angebotes, da für den Einkauf nun Auswahlmöglichkeiten bestehen.

- Für periodischen oder aperiodischen Bedarf angebotene Artikel werten ein Zentrum weiter auf, insbesondere dann, wenn auch sie mit Auswahlmöglichkeiten angeboten werden.

- Die Grundversorgung kann als gewährleistet betrachtet werden, wenn sämtliche Grundnahrungsmittel sowie gängige Genußmittel und einzelne Non-food-Artikel, wie sie in Lebensmittelgeschäften üblich sind, angeboten werden.
Tabelle 2: Gliederung der Subzentren von St. Gallen nach 3 Methoden

\begin{tabular}{|c|c|c|c|c|c|}
\hline & \multirow[t]{2}{*}{ Subzentrum } & \multicolumn{3}{|c|}{$\begin{array}{c}\text { Sedlacek }^{1)} \\
\text { Arbeitskräfte- } \\
\text { besatz }^{2)}\end{array}$} & \multirow{2}{*}{$\begin{array}{l}\text { - Zen- } \\
\text { trums- } \\
\text { stufe }\end{array}$} \\
\hline & & $\mathrm{Z}$ & $\mathrm{AB}$ & $\mathrm{AB}_{\mathrm{iG}}$ & \\
\hline 1 & Großacker & 11901 & 155 & (46) & 4 \\
\hline 2 & Lachen & 7753 & 101 & (58) & \\
\hline 3 & Neudorf & 7535 & 85 & (29) & \\
\hline 4 & St.Fiden & 5456 & 81.5 & (29) & \\
\hline 5 & Singenberg & 4359 & 64 & (15) & 3 \\
\hline 6 & Langgaße & 4180 & 39 & (19) & \\
\hline 7 & St.Georgen & 3091 & 58 & $(27))$ & \\
\hline 8 & Bruggen-Süd & 2842 & 36 & $(29)$ & \\
\hline 9 & Bhf St. Fiden & 2611 & 60 & (40) & \\
\hline 10 & Rotmonten & 2593 & 49 & (15) & \\
\hline 11 & $\begin{array}{l}\text { St. Othmar- } \\
\text { Oberstraße }\end{array}$ & 2006 & 26 & (16) & 2 \\
\hline 12 & Heiligkreuz & 1982 & 8 & (5) & \\
\hline 13 & Winkeln & 1944 & 16 & (13) & \\
\hline 14 & Krontal & 1522 & 41.5 & (11) & \\
\hline 15 & Riethüsli & 942 & 19 & $(12)$ J & \\
\hline 16 & Achslen & 497 & 4 & (4) & \\
\hline 17 & Zil & 372 & 3 & (3) & \\
\hline 18 & Schoren & 372 & 3 & (3) & 1 \\
\hline 19 & Bruggen-Nord & 372 & 3 & (3) & \\
\hline 20 & Hinterberg & 248 & 2 & (2) $)$ & \\
\hline
\end{tabular}

1) $\mathrm{z} \quad=\sum_{\mathrm{ij}} \frac{\mathrm{AP}_{\mathrm{j}}}{\mathrm{AP}_{\mathrm{i}}} \cdot \frac{1}{\mathrm{n}_{\mathrm{ij}}} \cdot \mathrm{f}_{\mathrm{i}} \cdot 1000$

2) $\mathrm{AB}=\mathrm{AP}_{j} \cdot \mathrm{m}$

$$
A B_{i G}=A P_{j} \cdot m_{i G}
$$

$\mathrm{AP}_{\mathrm{j}} \quad=$ Arbeitsplätze eines Geschäftes

$\mathrm{AP}_{\mathrm{i}}=$ Arbeitsplatzmittel der Branche $\mathrm{i}$

$\frac{1}{n_{i j}}$

$=$ Versorgungswert eines Geschäftes $\mathrm{j}$ der Branche $\mathrm{i}$

$\mathrm{f}_{\mathrm{i}} \quad=$ mittlere Frequenz der Branche $\mathrm{i}$

$\mathrm{m} \quad=$ Anzahl der Geschäfte im Subzentrum

$\mathrm{AB}=$ Arbeitskräftebesatz des Subzentrums = $=\mathrm{AP}_{\mathrm{j}} \cdot \mathrm{m}$

$\mathrm{AB}_{\mathrm{iG}}=\mathrm{AP}_{\mathrm{j}} \cdot \mathrm{m}_{\mathrm{iG}}$ Arbeitskräftebesatz der Branchen der Grundversorgung.

Dabei spielt die betriebliche Form des Angebots keine Rolle; die Grundversorgung kann gewährleistet sein durch eine Großverteilerfiliale oder durch eine vollständige Palette von kleinen Einzelhandelsgeschäften.

Diese Typisierung führt nun zu einer Gliederung der Subzentren, wie sie in Tabelle 2 vorliegt und lagegerecht in Abb.3 dargestellt ist. Wesentlich dabei ist, daß die Klaßenbildung nicht nach mathematisch-statistischen oder gar willkürlichen Überlegungen, sondern nach funktionalen Kriterien vorgenommen wird. 
Im Falle der Stadt St. Gallen (Tabelle 2 und Abb. 3) wird die Zentrumsstufe 4 nur erreicht vom Zentrum Großacker, das aus einem an das herkömmliche Quartierzentrum St. Fiden angelehnten integrierten Shopping Center mit 2 Großverteilerfilialen und 20 Detailgeschäften besteht. Dieses einzige höchstwertige Zentrum steht in einer gewissen Konkurrenz zum Hauptgeschäftszentrum der Stadt (vgl. auch Abb.2). In den sechs Subzentren der Zentrumsstufe 3 ist das über den Grundbedarf hinausgehende zusätzliche Angebot von Gütern des periodischen Bedarfs ansprechend; es wird in allen Fällen durch Güter für die aperiodische Bedarfsdeckung mehr oder weniger aufgestockt (vgl. Abb.2,3).

In sieben Subzentren der Stufe 2 wird die Zentrumsqualität durch vereinzelte oder mehrere Geschäfte mit einem speziellen Sortiment auf dem Niveau des periodischen und/oder aperiodischen Bedarfs aufgewertet; im Subzentrum Bahnhof St. Fiden wird sie von einer Großverteilerfiliale mit branchenübergreifendem Sortiment getragen.

Die fünf Zentren der untersten Stufe erreichen die Minimalbedingung der vollständigen Grundversorgung gerade knapp (Abb.2,3).

\subsection{Bestimmung von Versorgungsbereichen, Versorgungsqualität, Versorgungsgrad, Einzelhandelskapazität und A uslastungsgrad}

Mit dieser Reihe von Arbeitsschritten sind das Angebot der Subzentren (die Einzelhandelskapazität) und die flächenbezogenen und qualitativen theoretischen Nachfragemöglichkeiten der Versorgung im Stadtraum zu bestimmen. Schließlich ist der theoretische Auslastungsgrad der Subzentren durch die raumwirksam mögliche Nachfrage aus Versorgungsstandorten zu ermitteln.

\subsubsection{Versorgungsbereiche und \\ Versorgungsqualität}

Detaillierte wissenschaftliche und methodische Entwicklung dieses Arbeitsansatzes werden im Aufsatz von BOESCH (Lit.2) dargestellt und mit einer Anwendung auf die Stadt St. Gallen illustriert. Nachfolgend soll ein Überblick über die Idee und den Verfahrensablauf mit den wichtigsten Begriffserläuterungen gegeben werden:

Die Versorgungsbereiche umfaßen ein Gebiet, von dem aus die Bedarfsdeckung auf einer bestimmten Stufe im zugeordneten Subzentrum erfolgen kann (Abb.4).

Auf dem Niveau der Grundbedarfsdeckung z.B. sind die Versorgungsbereiche, zumindest bei traditionellem Einkaufsverhalten, durch die Erreichbarkeit im Rahmen von Fußgängerdistanzen determiniert, die für den mehrmaligen Einkauf pro Woche aufgewendet werden.

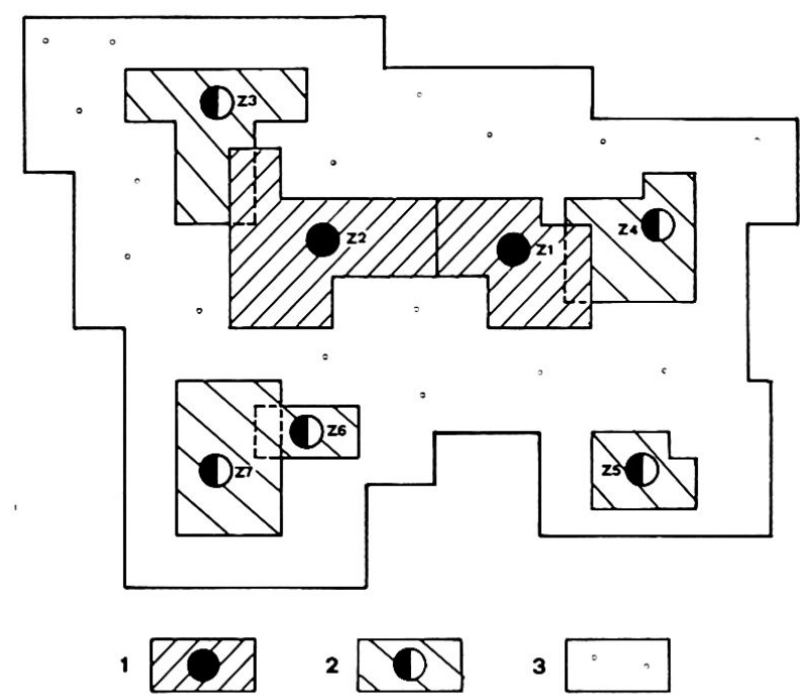

Abb. 4: Schematische Darstellung von Versorgungsbereichen, Versorgungsqualität und Versorgungsgrad auf dem Niveau der Grundversorgung

1 Versorgungsbereich der Subzentren (Z1/2) mit guter Versorgungsqualität

2 Versorgungsbereiche der Subzentren (Z3-7) mit genügender Versorgungsqualität

3 Stadtgebiet mit ungenügender Versorgungsqualität. Die Versorgung ist nur in Einzelläden oder Subzentren mit ungenügendem Angebot oder überdurchschnittlicher Distanzüberwindung möglich

Im vorliegenden theoretischen Fall ist eine zumindest genügende Grundversorgung für rund $40 \%$ des Stadtgebietes sichergestellt. Der Versorgungsbereich auf dieser Stufe könnte z. B. bei etwa 85\% der Stadtbevölkerung liegen.

Die Versorgungsqualität ist ein Maß für die Güte der Versorgung an einem bestimmten Konsumentenwohnort und auf einer bestimmten Stufe der Bedarfsdeckung.

Die Versorgungsqualität läßt sich mit den Gütestufen "gut, genügend, ungenügend» nach Angebot und Erreichbarkeit der Subzentren klassieren (vgl. Abb.4).

Für die Subzentrenanalyse von vordergründigem praktischem Interesse ist die Versorgungsqualität im Stadtraum auf dem Niveau des Grundbedarfs.

\subsubsection{Der Versorgungsgrad}

Die im Stadtraum - und für höhere Stufen der Versorgung im Stadtumland - ermittelten Versorgungsbereiche decken einerseits Flächen, andererseits auf diesen Flächen lokalisierte Bevölkerungsanteile ab.

Mit dem Versorgungsgrad soll nun ausgesagt werden, für welche prozentualen Anteile der Stadtbevölkerung die Minimalforderung der Grundbedarfsdeckung auf einem bestimmten Niveau der Versorgungsqualität erfüllt ist (vgl. Abb.4).

So könnten auf dem Niveau der Grundversorgung $25 \%$ der Fläche gut, $35 \%$ der Fläche genügend und $2 / 5$ der Fläche ungenügend versorgt sein. Der 
Versorgungsgrad wäre dann auf $60 \%$ der Fläche zumindest genügend und beträfe beispielsweise $90 \%$ der Einwohner (vgl. BOESCH, Lit. 2).

Hier ergibt sich ein Ansatz für stadtplanerische Überlegungen. Man wird sich als Ziel setzen können, daß der Versorgungsgrad für $100 \%$ der Bevölkerung und für das ganze bewohnte und in der Planung als Wohngebiet ausgeschiedene Stadtgebiet die Mindestanforderungen einer genügenden Grundversorgung erfüllen soll. Danach wären entsprechende Maßnahmen einzuleiten wie Ausbau oder Neuanlage oder zumindest planerische Sicherstellung von Subzentren.

Dabei könnten Bestandesaufnahmen über Abgrenzung und Ausstattung bestehender Subzentren (gemäß Abschnitt 3.1) weitere Entscheidungshilfen anbieten.

\subsubsection{Die Einzelhandelskapazität der Subzentren}

Wenn wir einerseits normative Forderungen für die Auslegung der Versorgungsbereiche gesetzt haben, müssen wir andererseits in Rechnung stellen, daß das bestehende Angebot eines jeden Subzentrums getragen werden muß von einer bestimmten Basisbevölkerung. Diese Basisbevölkerung ist im Idealfall mit der Bevölkerungszahl des theoretisch zugeordneten Versorgungsbereichs des Subzentrums identisch.

In der Arbeit von JETZER (Lit.9) werden Methoden zur Ermittlung der Einzelhandelskapazität vorgelegt und diskutiert.

Die Einzelhandelskapazität eines Subzentrums kann dabei errechnet werden in Anteilen seines Angebotes am Gesamtangebot des Stadtraumes außerhalb der City. Sie wird ausgedrückt in Angeboten an bestimmte potentielle Kundenzahlen (Basisbevölkerung). Die Einzelhandelskapazität kann der Bevölkerungszahl des Versorgungsbereiches eines Subzentrums genau entsprechen; sie kann größer oder kleiner sein als die vom Versorgungsbereichmodell gestellte Norm es verlangt (Abb.5).

Den Quotienten aus Einzelhandelskapazität (Basisbevölkerung eines Subzentrums) und der dem Subzentrum im Versorgungsbereich zugehörigen Bevölkerung bezeichnen wir als Auslastungsgrad.

Mit dem Auslastungsgrad der Subzentren können wir in erster Annäherung prüfen, ob die normativen Anforderungen der Erreichbarkeitskriterien für die Ermittlung von Versorgungsbereichen und Versorgungsgrad sinnvoll gesetzt oder allenfalls zu modifizieren sind.

Weiter lassen sich Thesen formulieren, mit welchen das Auftreten von Überkapazitäten (Auslastungsgrad $<1$ ) oder Unterkapazitäten (Auslastungsgrad $>1$ ) von Subzentren zu erklären versucht wird. Dabei ist wohl zu berücksichtigen, daß die Anziehungskraft eines Subzentrums aufgewertet

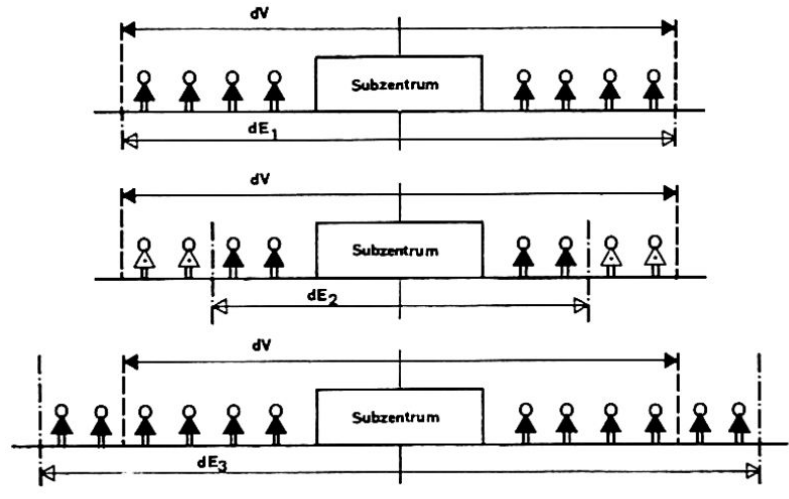

Abb. 5: Einzelhandelskapazität, Versorgungsbereich und Auslastungsgrad

$\mathrm{dV}=$ Versorgungsbereich des Subzentrums

$\mathrm{dE}_{\mathrm{n}}=$ Stadtgebiet, welches der Einzelhandelskapazität des Subzentrums entspricht

Fall 1: $d V=\mathrm{dE}_{1}$, Auslastungsgrad 1

Fall 2: $d V>d E_{2}$, Auslastungsgrad $>1$; die Einzelhandelskapazität vermag der Bedarfsdeckung im Versorgungsbereich nicht zu genügen. (Unterkapazität des Subzentrums)

Fall 3: $\mathrm{dV}<\mathrm{dE}_{3}$, Auslastungsgrad $<1$; die Einzelhandelskapazität des Subzentrums wird durch die Bevölkerung im Versorgungsbereich nicht ausgeschöpft. (Überkapazität des Subzentrums)

werden kann durch besonders attraktive Geschäfte, durch günstige Parkierungsmöglichkeiten oder durch leicht benützbare Poststellen und Bankfilialen. Die Stadtplanung hätte die Erhaltung oder Entfaltung solcher Attraktivitäten in Rechnung zu stellen und durch planerische Maßnahmen zu stützen. In andern Fällen können festgestellte Überkapazitäten in Zusammenhang stehen mit störendem Durchgangsverkehr oder dem Fehlen besonderer Attraktivitäten. Die Planung müßte sich bei solchen Verhältnissen für die Behebung behindernder Rahmenbedingungen oder für räumliche Neukonzeptionen entscheiden.

\subsection{Subzentrenattraktivität, Quartierstruktur und Einkaufsverhalten}

Die Anziehungskraft eines Subzentrums kann abgesehen vom Fächer der vorhandenen Ausstattung durch im vorstehenden Abschnitt genannte auf- oder abwertende Faktoren geprägt werden.

Von großer Bedeutung für das effektive Nachsuchen des Angebots durch die Bevölkerung des Versorgungsbereichs sind Quartierstruktur und Einkaufsverhalten.

In Quartieren mit eingesessener Bevölkerung besteht starke Tendenz zu traditionellem Einkaufsverhalten, nicht zuletzt auch wegen dem sozialen Kontaktverhältnis mit den Geschäftsinhabern.

Neubauquartiere erweisen sich als ausgesprochen subzentrumsunfreundlich bei hoher Mieterdurch- 
satzrate oder bei hohem Anteil doppelverdienender Ehepaare. Von ihnen wird der Wochenendeinkauf im kostengünstigsten Großverteiler oder der Kauf auf dem Weg zum oder vom Arbeitsplatz bevorzugt.

Durch Aufnahme von Bevölkerungsstrukturdaten (Alters-, Erwerbs-, Einkommens-, Haushaltgrößenstruktur u.a.) in den Quartieren (vgl. Lit. 1) sind für Planungsentscheide Hilfen zu erarbeiten. So dürfte es sich in bestimmten Fällen in Neubauquartieren als gegeben erweisen, die Anlage von nach normativen Gesichtspunkten erforderlichen Subzentren durch spezielle Bauvorschriften oder durch Festlegung wenigstens auf der Richtplanstufe offen zu halten.

Ein Erheben des effektiven Einkaufsverhaltens, in der Art wie sie von PoschwatTA (Lit.12, p. 200-202) bei 52 Haushalten eines Quartierteils der Stadt Augsburg vorgenommen hat, kann die Beurteilung des Aktualitätsgrads notwendiger planerischer Maßnahmen zugunsten von Subzentren verbessern helfen. Abgesehen vom fast unverhältnismäßigen Aufwand für solche Erhebungen, ist ihr langfristiger Prognosewert beschränkt. Stichprobenerhebungen in ausgesprochenen Problemquartieren werden sich jedoch lohnen, insbesondere dann, wenn sie in zeitlich relativ dichten Intervallen wiederholt werden können.

\subsection{Planerische Sicherung oder Freihaltung der Subzentren in den städtischen Richt- und Nutzungsplänen}

Es ist anzustreben, das Stadtgebiet für die Stufe der ausreichenden Grundversorgung mit einem Netz von Versorgungsbereichen mit zugeordneten Subzentren so abzudecken, daß ein möglichst hoher (wenn möglich hundertprozentiger) Versorgungsgrad erreicht wird. Im bereits überbauten Stadtgebiet sind diese Zentren in den Nutzungsplänen flächenmäßig zu fixieren, ihre Erhaltung oder ihr Ausbau mit Zentrumsbauvorschriften zu ermöglichen oder zu erleichtern.

In geplanten städtischen Wohngebieten sind die nach dem normativen Modell der Versorgungsbereiche erforderlichen neuen Subzentren zumindest in den Siedlungsrichtplänen festzulegen.

\subsection{Dauernde Überwachung und periodische Datenüberprüfung}

Sowohl die Mutationen in den Subzentren als auch die Änderungen in der Struktur der Quartierbevölkerung sind dauernd $\mathrm{zu}$ überwachen und $\mathrm{zu}$ registrieren, Bevölkerungsdaten im Fünfjahreszyklus oder zumindest in den Spannen der Eidg. Volkszählung alle 10 Jahre neu aufzuarbeiten. In manchen Bereichen kann erst mit der zeitlichen
Dimension und mit der Bewußtmachung von städtischer Dynamik die Prognosesicherheit verbessert werden.

\section{Anmerkungen}

1) Die Begriffsbestimmung entspricht jener von WOLF (Lit. 15), der «Subzentrum» und «Nebenzentrum» gleichbedeutend verwendet. Dagegen ist unser Begriff Subzentrum umfaßender als bei ABELE/LEIDLMAIR (Lit. 1), die ihn einschränkend für die höchstwertigen Zentren außerhalb des Hauptgeschäftszentrums verwenden.

2) Der Versorgungswert drückt aus, wieviele Einwohner des Stadtgebietes außerhalb der City im Mittel von einem Geschäft einer bestimmten Branche versorgt werden (Lit. 14, p. 24).

3) Diesem Faktum wurde auch von SEDLACEK (Lit.14, p.25) Rechnung getragen durch branchenweises Berechnen des Arbeitsplatzbesatzes.

4) In größeren Städten mit mehreren Subzentren auf citynahem Niveau kann das Schema durch qualitative Anforderungen bezüglich der Versorgung mit Gütern des periodischen und aperiodischen Bedarfs erweitert werden.

\section{Résumé}

\section{La problématique relative à la recherche dans le domaine de la centralité intraurbaine}

Parmi les objectifs de la recherche classique et celle de date plus récente dans le domaine de la centralité intraurbaine, celui visant à l'élaboration de hiérarchies à plusieurs degrés occupait une place prédominante. La hiérarchisation du réseau des centres intraurbains s'est effectuée en particulier sur une base formelle et descriptive, soit d'une manière empirique (PROUDFOOT, BURNS), soit à l'aide d'indicateurs (JONES, GARNER et autres) ou sur le fond d'une approche quantitative (SEDLACEK). C'est CAROt qui abait déjà relevé l'importance des fonctions des centres secondaires dans le contexte urbain.

Sur le plan de l'aménagement urbain il s'agit avant tout de préciser les fonctions des centres secondaires dans le cadre du processus d'approvisionnement. C'est dans cette perspective qu'un procédé est envisagé se composant des phases de travail suivantes:

1. identification et délimination des centres intraurbains, ainsi que leur différenciation et pondération en fonction des catégories de biens de consommation.

2. détermination de la qualité d'approvisionnement, des zones d'approvisionnement et du 
degré d'approvisionnement relatif à l'espace urbain; détermination de la capacité de l'équipement commercial et du taux d'expoloitation des centres intraurbains.

3. analyse des centres intraurbains et de leurs zones d'approvisionnement en fonction de l'attractivité des centres, de la structure socioéconomique des quartiers résidentiels et du comportement actuel des consommateurs

4. prescriptions d'aménagement concernant le maintien ou la sauvegarde de zones libres de constructions (destinées à l'implantation de services centraux) dans les plans directeurs et d'affectation urbains

5. surveillance permanente et contrôle périodique des données en vue de revisions possibles ou nécessaires des plans d'affectation spatiaux.

La première phase de travail est commentée et illustrée à l'aide d'exemples sur la base d'enquêtes menées en ville de Saint-Gall. Le procédé méthodique de la deuxième phase de travail est présenté dans les travaux de BOESCH et JETZER, travaux qui ont été réalisés simultanément, sous la conduite de l'auteur, à l'Institut pour la Géographie Economique et l'Aménagement du Territoire à l'Ecole des Hautes Etudes Economiques et Sociales de Saint-Gall.

\section{Zitierte Literatur}

1) ABELE G., Leidlmair A.: Karlsruhe. Studien zur innerstädtischen Gliederung und Viertelsbildung. Karlsruher Geogr. Hefte 3, 1972.

2) BOESCHM.: Versorgungsbereiche, Versorgungsqualität und Versorgungsgrad am Beispiel der Detailhandelsversorgung der Stadt St.Gallen. Geogr. Helv. 35, 2, 1980, p. 59-66.

3) BugMANNE., BOESCHM., JETZER J.-P.: Das Geschäftszentrum von St.Gallen. Geogr. Helv.31, 1976, p. 167-172.

4) BURNSW.: British shopping centres. London, 1959.

5) CARTER H.: The Study of Urban Geography. Arnold London, 1972.

6) CAROLH.: The hierarchy of central functions within the city. Annals, Ass.Am. Geogr. 50, 1960, p. 419-438.

7) CHRistaller W.: Die zentralen Orte in Süddeutschland. Jena 1933.

8) GARNER B. J.: The internal structure of retail nucleations. Northwestern Univ. Stud. Geogr. 12, 1966.

9) JETZER J.-P.: Capacité des équipements commerciaux et taux d'exploitation des Centres d'Ap- provisionnement Intraurbains. Geogr. Helv. 35, 2, 1980, p. 67-75.

10) JOHNSTONR.J.: The distribution of an intrametropolitan central place hierarchy in Melbourne. Aust. Geogr. Stud. IV/1966, p. 17-33.

11) JONES R.: Central place theory and the hierarchy and location of shopping centres in a city. Inst. Br. Geogr. Study Group in Urban Geography, Edinburgh 1967.

12) POSCHWATTA W.: Verhaltensorientierte Wohnumfelder. Geogr. Rundschau 30, 1978, p. 198205.

13) PROUDFOOTM.J.: City retail structure. Economic Geography 13, 1937, p. 425-428.

14) SEDLACEKP.: Zum Problem intraurbaner Zentralorte, dargestellt am Beispiel der Stadt Münster. Selbstverlag Geogr. Komm. Münster/ Westfalen, 1973.

15) WOLFK.: Stadtteil-Geschäftsstraßen. Ihre geographische Einordnung, dargestellt am Beispiel der Stadt Frankfurt am Main. Frankfurt a.M. 1969.
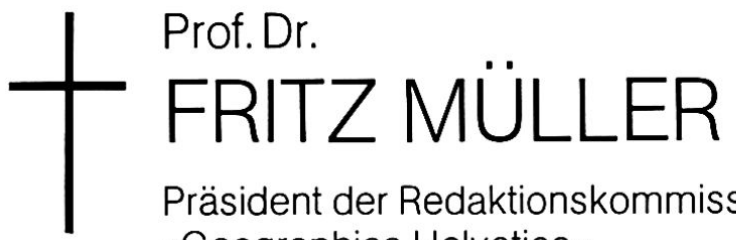

Präsident der Redaktionskommission "Geographica Helvetica" Präsident der «GeographischEthnographischen Gesellschaft Zürich»

Der Vorsteher des Geographischen Instituts der ETH Zürich, Prof. Dr. Fritz Müller, ist am späten Samstagvormittag, den 26. Juli 1980, auf einer Seitenmoräne des Rhonegletschers, während einer Exkursion mit Gemeinde- und Pressevertretern, die der Darlegung seiner Forschungsarbeiten galt, einer Herzkrise erlegen.

Eine ausführliche Würdigung seiner wissenschaftlichen Tätigkeit folgt im Heft 3 der "Geographica Helvetica».

Die Redaktion 